\title{
The Discovery of Floating Boats on the Oscillating Levitated Liquid Layer
}

\author{
Angela Li', and Danny Zhang ${ }^{1}$ \\ ${ }^{1}$ Exploring Scholar, North York, ON, CA \\ DOI: https://doi.org/10.47611/jsrhs.v10i2.1496
}

\begin{abstract}
$\underline{\text { ABSTRACT }}$
When air bubbles are injected into a still bath with liquid, it's known that they will rise to the top surface because of their small mass. However, oscillations, which can provide a dynamical stabilizing effect, can make air bubbles sink when created below a certain depth that changes along with the forcing amplitude. Three experiments are needed to show the defy-gravity behavior caused by vibration on liquid. Using silicon oil, a syringe, a needle, and a vertically oscillating shaker with amplitude, an air layer formed by sinking bubbles, which defy the well-known Archimedes' principle, is being trapped under the levitating liquid layer acts as a spring-mass. With the further experiment using two light foam boats, it was observed that an upside-down buoyant force was acting on the liquid provided by vibration, supporting the boats to flow on both interfaces of the liquid layer. A symmetric Archimedes' principle is discovered to be reflected on the lower interface created by the dynamic stabilization as well. This discovery about the effect vibration can have on liquid, and alternately the forces acting on the object floating on this liquid brings lots of future development to light and is new progress in the area of fluid mechanics.
\end{abstract}

\section{Introduction}

On September 2, 2020, a research paper about "Floating under a levitating liquid" written by 4 scientists, Benjamin Apffel, Filip Novkoski, Antonin Eddi, and Emmanual Fort, from ESPCI was published in Nature ${ }^{[1]}$ This paper attracted huge attention from the public, with over 16k accesses in 3 months and relative news articles written in more than 10 languages, including English, French, Mandarin, etc. So, what exactly was their discovery that attracted such great attention?

We are all familiar with how the gravity and buoyant force are acting on an object that's floating in a still bath, as well as the property that objects with more density sink. But what if an external force (vibration) was being put upon the object or this still bath as a whole? This was when a phenomenon that we wouldn't normally see occur. A pendulum would stay up straight without falling due to gravity because of vibration, ${ }^{[2]}$ so will vibration also have an effect on liquid? How can this observation be used to discover something more astonishing?

In this paper, I discuss in detail some physics concept about the area of gravity and buoyancy, and the important background information needed before pursuing the experiment, continue on to the procedure of three experiments conducted by ESPCI's scientists along with some analysis and finish off focusing on the future development and applicant this discovery can possibly bring. 


\section{Context}

Buoyancy, also known as the buoyant force, was the upward force exerted on an object that was fully or partially immersed in a fluid.[3] This force explains how a boat conquers the force of gravity and was able to float. The science behind this has remained mainly unchanged since ancient Greek mathematician Archimedes discovered a physical law of buoyancy, known as Archimedes' principle. Archimedes' principle states that any object immersed in a fluid at rest, either completely or partially, was acted upon by an upward force, buoyant force.[4] This buoyant force was equal to the weight of the fluid being displaced, which was equivalent to the volume of the object. This principle suggests how the downward force of gravity was balanced by upward pressure from the displaced water. Gravity, a downward force that was opposite to buoyant force, was discovered by Isaac Newton when he saw a falling apple while thinking about the forces of nature.[5] According to Archimedes' principle and further study build on to it, if an object flows on the fluid, then it means the buoyant force acting on to the object was greater than the weight of it, which was equal to its mass times the downward acceleration caused by gravity. And if the object sinks, then it's the opposite. The relationship between the two forces allows activity and certain phenomenon to occur. Surfing was an example, where the force of gravity cancels out with the upward force exerted by the water onto the contacting surface of the board which helps the surfers to maintain balance.

Before continuing studying about the levitating fluid, it's necessary to understand the Kapitza effect that was supporting it. The Kapitza effect shows an unusual equilibrium feature of the pendulum under large frequency and the amplitude of the pivot vibrations. ${ }^{[6]}$ The suspension point of a simple rigid pendulum was forced to vibrate at a high frequency along the vertical line. Vibration keeps the pendulum balance upright rather than swing back down to its natural position due to the force of gravity, showing the dynamic stabilization of the inverted position. ${ }^{[2]}$ This surprising phenomenon of dynamic stabilization was predicted originally by Stephensons in $1908,{ }^{[7]}$ but no explanation and further analysis had been made. And in 1951, this extraordinary phenomenon was rediscovered, along with physical explanation and experimental investigation in detail by Pyotr Kapitza, a Nobel prizewinning physicist. ${ }^{[8]}$ It was later named after this great physicist. Kapitza explained in the paper published in the Russian journal that "Our eyes cannot follow the fast small movements caused by vibrations of the pivot, so the behavior of the pendulum in the inverted position seems perplexing and even astonishing.... When we carefully touch the rod of the pendulum trying to deviate it from the vertical, the finger feels the resistance produced by the vibrational torque." ${ }^{[7]}$ The Kapitza effect attracts huge attention from scientists around the world and was hoped to have further discovery and progress built onto what Kapitza had found.

With further investigation by scientists since then, the phenomenon of the levitating liquid has been discovered. Using the vertically oscillating shaker with amplitude, scientists can make liquid levitate in midair and let air bubbles sink rather than rise. ${ }^{[9]}$

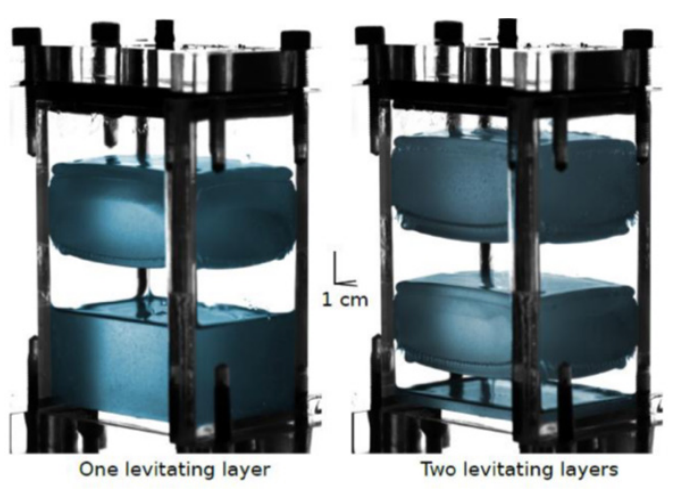

Figure 1. Levitating Liquid Layers. Oscillating containers with one and two levitating liquid layers of silicone oil. 
The image shows one levitating layer with part of the fluid slowly dripping down while the image on the right shows how both layers of liquid can be levitated by increasing the frequency of vibration. These experiments helped support the newest discovery about small boats flowing above and under the levitating liquid layer ${ }^{[10]}$ that seemed to defy gravity and our previous knowledge about buoyancy. The new discovery that challenged our intuitive understanding of liquid-air interfaces that could possibly prompt future investigations of the behaviors of liquid boundaries were found by a group of scientists from ESPCI in Paris, who was inspired by the Kapitza effect, and hoped to employ the phenomenon of defy-gravity by vibration on solid to liquid.

\section{Experiment}

\section{Experiment 1: Sinking bubbles in a shaken liquid bath ${ }^{[11]}$}

Before further investigation about the levitating liquid and upside-down boats, an experiment about "Sinking bubbles in a shaken liquid bath" ${ }^{[11]}$ was conducted by the scientists from ESPCI. The bubbles were used to create an air layer trapped below the levitating liquid layer. To let bubbles, stay below the liquid layer, they needed to sink to instead of rising to the upper surface. This defy-standard buoyancy behavior could be achieved by the oscillation.

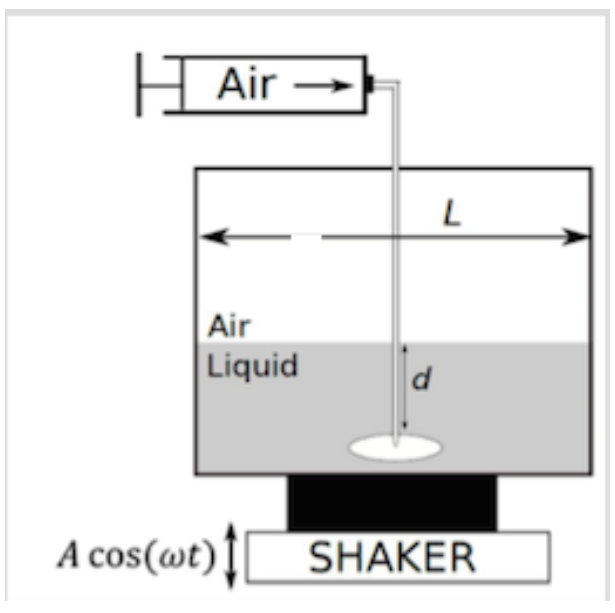

Figure 1A. Experimental Setup Schematic. Experimental setup composed of a Plexiglas container of various sizes attached on a vertically oscillating shaker. The liquid was either glycerol or silicon oil. The bubbles were created by injecting air with a syringe through a long needle.

The experiments were operated in a room with a constant room temperature of $20 \mathrm{C}^{\circ}$. The experimental setup could be modeled using a schematic (see figure 1A). Plexiglas containers with different side lengths (up to $20 \mathrm{~cm}$ in width) were being prepared. A Plexiglass container was being attached to a "vertically oscillating shaker with amplitude $A$ and frequency $w / 2 \pi$ " ${ }^{[11]}$ The liquid was shaken at a frequency of $100 \mathrm{~Hz}$. The liquid being used in the experiment was either glycerol or high-viscosity silicone oil. A syringe and a long needle were being used to inject air into the liquid, creating the bubbles. 
The various depths the air bubbles were created at using the long needle was represented as $d$. Without the oscillations provided by the shaker, the buoyant force put onto the bubbles were much greater than the gravitational force, so the bubbles would not sink. But with the oscillations, the collective forces acting on the bubble created an overall downward net force which pushed the bubble down, resulting in the sinking of the bubbles. ${ }^{[12]}$

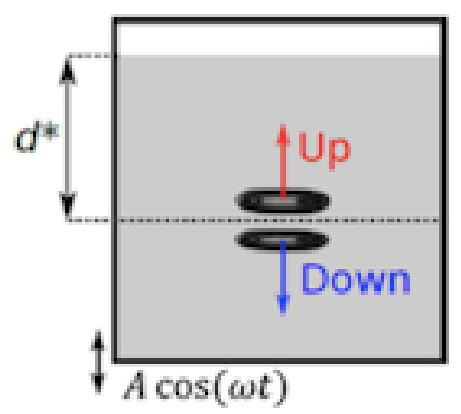

Figure 1B. Motion of Bubbles Schematic. Bubbles produced above and below the critical sinking depth $d^{*}$.

With the oscillation, bubbles were observed to sink when created below $d^{*}\left(d>d^{*}\right)$ and raised to the top if created on top of $d$ (see figure 1B). However, the range of value $d^{*}$ wasn't always constant. It changed as the shaking amplitude A increased (see figure 1C below). Fig.1C shows $d^{*}$ as a function of the shaking amplitude $A$. Modeled data were calculated and fitted by an invert quadratic scaling $c / A^{2}$ where $c$ had the value of $5.5 \mathrm{~mm}^{3}$. Two insets are provided in the figure showing the change in range of value $d^{*}$. When the value of shaking amplitude was around 0.475 (the inset on the left in figure 1C), the range of $d^{*}$ was clearly wider than the range below $d^{*}$. According to Fig.1B, the bubbles above $d^{*}$ would rise to the top surface, only the bubbles below the critical sinking depth $d^{*}$ would sink. So in the first case, more bubbles would rise than sink. The second inset illustrates how the critical depth $d^{*}$ started to sink decreases when the forcing amplitude increased.

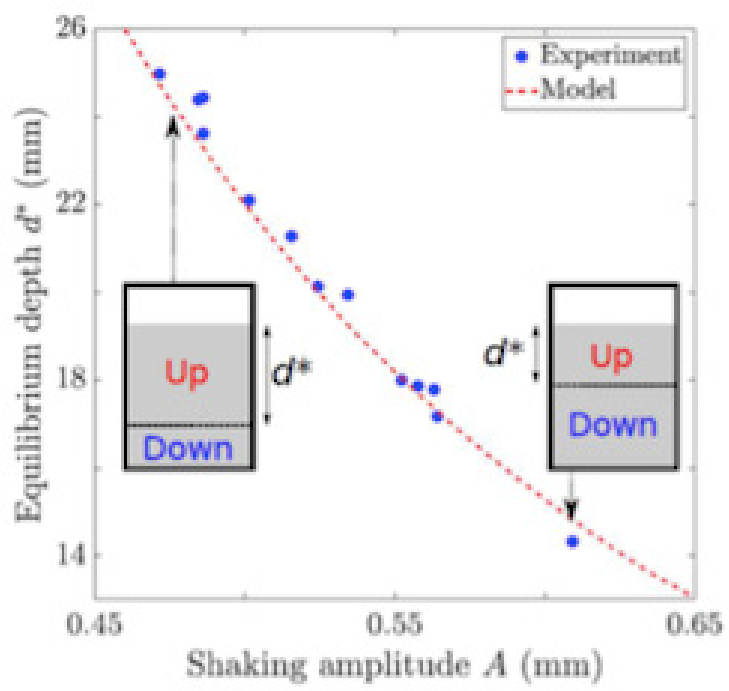

Figure 1C. Shaking Amplitude $A$ - Equilibrium Depth $d^{*}$ Graph. Evolution of the critical sinking depth $d^{*}$ as a function of the shaking amplitude $A$. Data were fitted by an invert quadratic scaling $c / A^{2}$ with $c=5.5 \mathrm{~mm}^{3}$. 
The experimental data in Fig.1C shows $d^{*}$, as a function of the shaking amplitude $A$, would be modeled better and more accurately using an adjusted inverted quadratic scaling $1 / \mathrm{A}^{2}$, instead of $c / A^{2}$ where $c=5.5$ as predicted. With the illustration presented by Fig.1B along with graph $1 \mathrm{C}$, the conclusion that more bubbles would sink as shaking amplitude increased could be obtained. Having an air layer trapped below the levitating layer created by these bubbles was essential to successfully levitate the whole liquid layer, that's why it was necessary to understand the way to control these bubbles, which was by adjusting the shaking amplitude as concluded in this experiment.

\section{Experiment 2: Levitating liquid layer stabilized by Kapitza effect ${ }^{[13]}$}

Inspired by the Kapitza effect, ESPCI's scientists reproduced the similar defy-gravity phenomenon on liquid instead of solid, showing how strong vibration could keep the downward pull of gravity and upward pull of buoyancy balanced.

This experiment was a continuation of experiment 1 which focused on creating the air layer. The experimental setup was the same as in experiment 1 with the requirement of the following materials: Plexiglas containers in various sizes, a vertically oscillating shaker, glycerol or high-viscosity silicone oil, a syringe, and a long needle. The liquid used in this experiment operated by the ESPCI scientists was the silicone oil, vibrating in the shaker with the forcing frequency of $100 \mathrm{~Hz}$. The initial forcing amplitude of $3 \mathrm{~mm}$ but was slowly decreased after the levitating layer was formed.

To make the liquid layer levitate, creating an air layer by blowing air at the bottom of the oscillating liquid bath using the needle was the first step. As being concluded in experiment 1, vibration can cause the sinking of the bubbles until the bottom of the bath was being completely filled, creating an air layer trapped below the levitating liquid layer. The trapped air layer was prevented from being released by the lower interface of the levitating liquid, which was stabilized by the vertical shaking. "The vertical vibrations have a stabilizing effect on the lower fluid interface. This can be interpreted as a Kapitza effect which consists of dynamical stabilization of an inverted pendulum by vertical shaking." [11]

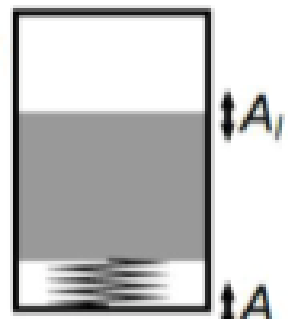

Figure 2A. Spring-mass Systems Schematic. Schematics of the spring-mass systems composed of the air layer loaded with the levitating liquid.

The schematic of the "spring-mass systems composed of the air layer loaded with the levitating liquid" was presented (see figure 2A). To make the liquid layer levitate, this air layer was needed to "act as a vertical springloaded with the liquid mass placed upon it and driven by the shaker". ${ }^{[11]}$ The initial forcing amplitude provided by the shaker was $3 \mathrm{~mm}$ and was decreased after the levitating layer was formed. The reason for this decrease of forcing amplitude was to prevent the Faraday instability on both sides of the fluid layer that could be excited near the resonance when the vibration frequency exceeded the critical value, causing the instability of the flat hydrostatic surface. ${ }^{[14]}$ After successfully levitated a liquid layer, the scientists from ESPCI investigated the relationship between the length of the container and the threshold excitation velocity $A l w$ for Kapitza stabilization of the liquid layer. 


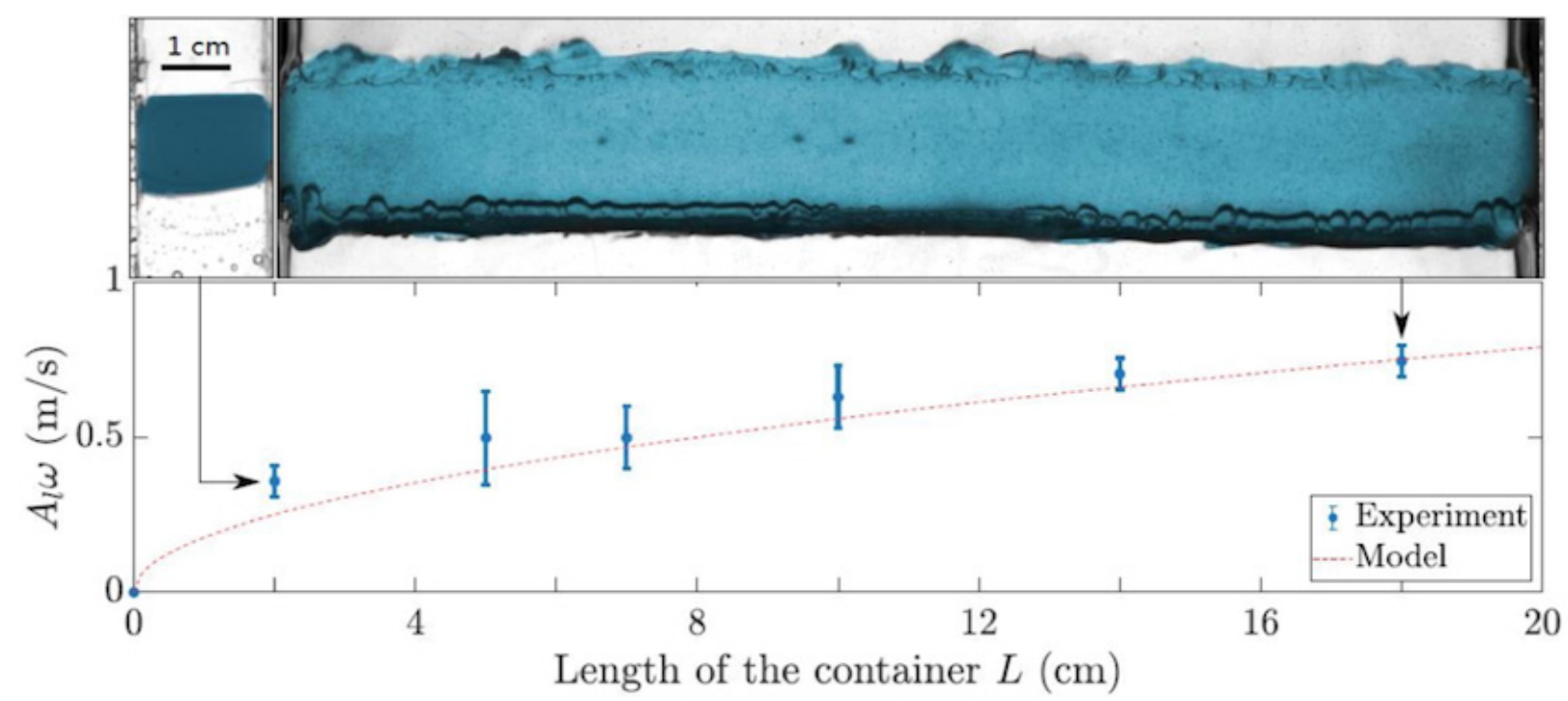

Figure 2B. Length of Container - Alw Graph. Threshold excitation velocity $A 1 w$ for Kapitza stabilization of the liquid layer as a function of the length $L$ of the container: experimental data (circles) and model $A 1 \mathrm{w}=\sqrt{ } g L / \pi$ (dashed line). Above are side views of the levitating bath in a $2 \mathrm{~cm}$ and $18 \mathrm{~cm}$ wide container

The modeled data was calculated and fitted by the formula $A l w=\sqrt{ } g L / \pi$, while the circles represented the experimental data (see Fig.2B). "The liquid being used was silicon oil. The forcing frequency was constant and tuned to stay at $80 \mathrm{~Hz}$." ${ }^{[15]}$ The graph shows the threshold excitation velocity Alw for Kapitza stabilization of the liquid layer as a function of the length $L$ of the container, providing the oscillating velocity $A l w$ needed to stabilize baths with lengths $L$ up to $18 \mathrm{~cm}$. The formula of the interface stability $(A l w>\sqrt{ } g L / \pi)$ was obtained by the limit to the observed excitable wavenumber $k>2 \pi / L$ sets by the limited size $L$ for the bath. Two insets show views of the levitating layers when the inputs $L=2 \mathrm{~cm}$ and $L=18 \mathrm{~cm}$. These insets show how the interfaces of the levitating liquid layers were more stable when $L=2 \mathrm{~cm}$ than when $L=18 \mathrm{~cm}$. This doesn't mean the instability would always increase as the length of the container increases, but meaning the shaking amplitude, as well as the viscosity of the liquid, must always be high enough to prevent Faraday instability. The graph clearly shows how the threshold excitation velocity Alw for Kapitza stabilization of the liquid layer was increasing as the length of the containers increase, thus we can get the conclusion that the second way to control the stability of the levitating layer (other than changing the shaking amplitude) was to change the sizes of the containers. This was a piece of important information to gain and understand for the benefit of conducting the third experiment, focusing on relative stability between a floater and the liquid layer.

\section{Experiment 3: Archimedes' principle over and under a levitating liquid layer [11][13]}

The observation made by scientists from ESPCI in the first two experiments wasn't new. Some scientists had discovered the levitating liquid layer supported by the Kapitza effect already. The conclusion and explanation were provided, that the strong vibration can cause the viscous fluids to hover, because of the opposing force vibration provided to the fluid that pushes the fluid back up and stops the lower surface of fluid from breaking up. ${ }^{[16]}$ However, what the scientists from ESPCI discovered and concluded in this experiment was totally new, showing how the development of technology was challenging some of our previous and current knowledge. This experiment was a build-on to experiment 1 and 2, to further investigate and analyze the applicant of the observation made in those experiments. 
In this experiment, the scientists from ESPCI focused on the possibility of having objects floating at both surfaces of the levitating fluid layer, which means an object needed to flow on the inverted interface of the layer upside-down. A schematic was provided to show how the buoyant force and the weight of the immersed bodies cancel each other to stay balanced at two opposed interfaces of the levitating fluid layer (see figure $3 \mathrm{~A}$ below). This could be simply redone by using the method mentioned in experiment 2 to levitate a liquid layer and put two identical objects on both interfaces of the liquid layer. With the explanation of Archimedes' principle, the upper, as well as the lower interface, exhibited an upward buoyant force equals to the weight of displaced liquid.

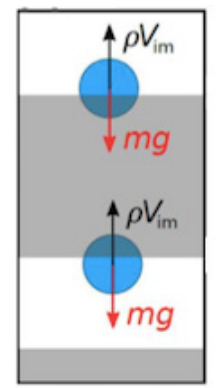

Figure 3A. Buoyant and Gravity Force Schematic. Schematics of the force balance at the two opposed interfaces with buoyant force cancelling the weight of immersed bodies.

To further investigate how buoyant force was acting on these objects, a graph showing the typical potential exerted on the objects without considering the dynamic effect was being used by the scientists (see figure 3B).

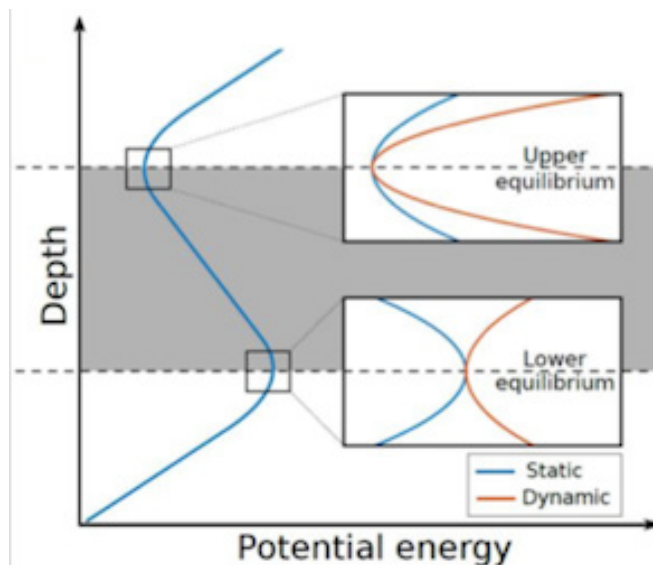

Figure 3B. Potential Energy - Depth Graph. Two equilibrium positions appear at each interface, the lower one being unstable. 
One equilibrium position was spotted on each interface, while the upper equilibrium was stable, and the lower equilibrium was not. If an object was placed on the lower interface, it could easily fall if it was being pushed downward and could float back on the upright interface if the buoyant force was too big. But with the oscillation or taking the dynamic effect into account, an extra stabilization was added to both equilibrium positions. The vibration here not only provided the lower horizontal interface stability, but also enhanced the vertical stabilization of the unstable equilibrium position. This was a dynamical anti-gravity behavior, which was the reason why boats were able to float on both interfaces (see figure $3 \mathrm{C}$ ).

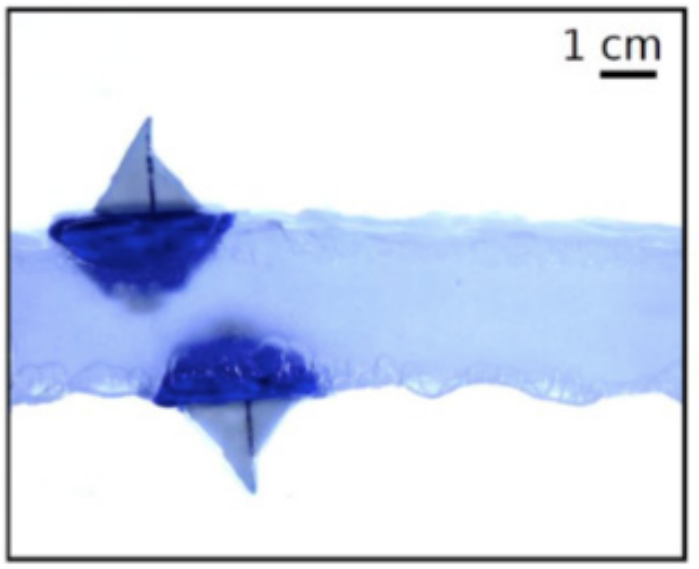

Figure 3C. Upside-down Boat. Boats floating over and under a levitated liquid layer.

In this "upside-down floating boats" experiment done by the ESPCI's scientists, the liquid used was the silicone oil in the container with the horizontal section of $14 \times 2 \mathrm{~cm}^{2}$, shaking under the forcing frequency of $60 \mathrm{~Hz}$. ${ }^{[15]}$ The two light foam boats had a dimension of $1.5 \mathrm{~cm} \times 3 \mathrm{~cm}$. It was observed that with the vibration, the boat on the lower surface could stay perfectly balanced with the net force of zero, where the downward pull of gravity canceled out with the upward pull of buoyancy.

\section{Further Studies and Application}

The discovery found in these conducted experiments about the effect vibration can have on liquid are significant in terms of the development of the area of buoyancy and the field of fluid mechanics in physics. Not only the conclusion gets from this experiment can be used in the future as a method of control the motion of bubbles for extraction or mineral processing, ${ }^{[16]}$ but by knowing how vibration can challenge our intuition and current knowledge through this one experiment can attract more attention from scientists around the world to employ the uses of vibration on other experiments to discover any exotic behaviors. As concluded in these experiments, vibration can cause the gravitydefy behavior built on to a phenomenon (liquid can levitate in the air while bubbles sink to the bottom) that's contradicting some of our previous knowledge about buoyancy that denser objects sink while the object with fewer density floats. With this discovery, vibration can be used in the activity of extracting liquid (ie.oil) from gas or the activity of mineral processing by controlling the motion of bubbles to let them sink instead of rising to the top surface covering the upper interface of the liquid.

One of my personal suggestions regarding the further study of this topic was to change the liquid being used in the experiment possibly along with the change of temperature. The liquid being used in this experiment was high viscosity silicone oil, and it's stated that viscosity was the only constraint in this experiment. (volume of the liquid was a limiting factor but can be easily solved by simply increasing the forcing amplitude and frequency) Water or oil isn't viscous enough to levitate in the container, but something like syrup might possibly work. Syrup might not be 
viscous enough to levitate if the temperature was high, since the viscosity of the liquid decreases as temperature increases. So it's possible that if the room temperature was being decreased, then more liquid can by employ in the experiment (but not too low that it freezes)

This was a new observation made by ESPCI's scientists from 3 months ago, so it would be beneficial to repeat the same process by another to ensure the accuracy of the data and the conclusion. With the same method, a different angle or direction might be focused on by the other team, so a new observation can possibly be made. Built onto the procedure and material used in this experiment, a further investigation was suggested. In this experiment, to investigate how the boat can flow upside-down on the lower interface of the levitating liquid layer, the scientists used a foam boat with little mass that won't be easy to fall down because of the huge weight (gravity). So, if the experiment can be successfully redone again by another team, then changing the mass or the material of the boat can be a good extension to start with.

People who can be benefited from learning more about this topic are not limited to any community group. Since the original research paper was an educational physics paper that's deep in depth, it's probably more beneficial for university students to read. But in terms of the topic, everybody can be benefited from learning more about it. More specifically, the scientists working or are interested in the area of buoyancy, gravity and are hoping to discover any new exotic behaviors by applying the knowledge and uses of vibration.

\section{Conclusion}

The experiments conducted by ESPCI's scientists wouldn't be possible without the previous discovery found by all the great physicists and mathematicians. It had been a long road to the discovery of the upside-down boat floating under the levitating liquid layer. From Archimedes' principle, the fundamental law of physics discovered by Archimedes, to Gravity, one the greatest discovery found by Newtons ${ }^{[5]}$ From Kapitza pendulum effect, the first time the method vibration was being used to conquer the gravity force acting on solid discovered by Nobel Prize-winning physicist Kapitza, ${ }^{[7][8]}$ to these outstanding phenomenon observed by four great scientists from ESPCI.

Using all this previous knowledge, ESPCI's scientists concluded that the motion of the bubbles can be controlled by the forcing amplitude and frequency, forming an air layer at the bottom of the bath, and with oscillation added to the system, a high viscosity liquid layer with two foam boats floating on both interfaces can be levitated. While the volume of the liquid and the size of the container can be limited due to the maximum forcing amplitude and frequency of the shaker, the viscosity of the liquid seems to be the only constraint throughout the whole experiment. ${ }^{[1]}$

This great discovery about the effect vibration has on liquid brings lots of potential future development to light. From extracting liquid from gas to mineral processing, from surfing upside-down to conquer the gravity force put upon gas, these could all be benefitted or possible to achieve with the discovery about vibration in these experiments. With these experiments showing a defy-gravity behavior that was challenging our intuition and current knowledge, more scientists, or simply people from the public, can be inspired to employ the uses of vibration on other experiments to discover any exotic behaviors.

\section{Acknowledgements}

I would like to express my deep gratitude to the authors of the research paper "Floating under a levitating liquid", Benjamin Apffel, Filip Novkoski, Antonin Eddi and Emmanuel Fort, for the experiments they conducted, and the insightful analysis comes along with the graphs and schematics they made. My paper wouldn't be possibly written without their works. Special thanks should be given to Mr. Danny Zhang, my research supervisor, for his patient guidance, useful critiques, and valuable assistance throughout my whole paper writing processes. I would also like to 
extend my thanks to Elaine Li for her encouragement and advice. Finally, I wish to thank my parents for the insightful discussions and support throughout my whole study processes.

\section{Bibliography}

[1] J. Walter, Discover Magazine (2020).

[2] P.H. Jones et al, Search the World's Largest Collection of Optics and Photonics Applied Research. (2016).

[3] The Editors of Encyclopaedia Britannica et al, Encyclopaedia Britannica (1998).

[4] Khan Academy.

[5] A. May, Sciencing (2018).

[6] C.J. Richards et al, Nature News (2018).

[7] E.I. Butikov, Kapitza Pendulum: A Physically Transparent Simple Explanation 1 (n.d.).

[8] NobelPrize.org.

[9] I. Sample, The Guardian (2020).

[10] Emmanuel Fort Professeur de ESPCI Paris and Benjamin Apffel Doctorant, The Conversation (2020).

[11] B. Apffel et al, Nature 3 (2020).

[12] C. Gentry et al, Sinking Bubble in Vibrating Tanks 5 (n.d.).

[13] B. Apffel et al, Nature News (2020).

[14] G. Pucci, International Journal of Non-Linear Mechanics (2015).

[15] B. Apffel et al, Nature News (2020).

[16] E. Gent et al, Science (2020).

[17] K. Chang, Baltimoresun.com (2020).

\section{Figures:}

"Levitating Liquid Layers": https://arxiv.org/pdf/2003.04777.pdf

"Experimental Setup Schematic" (figure 1A): https://arxiv.org/pdf/2003.04777.pdf

"Motion of Bubbles Schematic" (figure 1B): https://arxiv.org/pdf/2003.04777.pdf

"Shaking Amplitude $A$ - Equilibrium Depth $d^{*}$ Graph" (figure 1C): https://arxiv.org/pdf/2003.04777.pdf

"Spring-mass Systems Schematic" (figure 2A): https://arxiv.org/pdf/2003.04777.pdf

"Length of Container - Alw Graph" (figure 2B): https://arxiv.org/pdf/2003.04777.pdf

"Buoyant and Gravity Force Schematic" (figure 3A): https://arxiv.org/pdf/2003.04777.pdf

"Potential Energy - Depth Graph" (figure 3B): https://arxiv.org/pdf/2003.04777.pdf

“Upside-down Boat" (figure 3C): https://arxiv.org/pdf/2003.04777.pdf 